\title{
Rapidly Progressive Pituitary Apoplexy in a Patient with COVID-19 Disease Treated with Endoscopic Endonasal Surgery
}

\author{
Charit Taneja, MD ${ }^{1}$ Pouneh K. Fazeli, MD, MPH ${ }^{1}$ Paul A. Gardner, MD ${ }^{2}$ Eric W. Wang, MD \\ Carl H. Snyderman, MD, MBA ${ }^{3}$ Hussain Mahmud, MD $^{1}$ \\ ${ }^{1}$ Division of Endocrinology and Metabolism, University of Pittsburgh \\ Medical Center, Pittsburgh, Pennsylvania, United States \\ 2 Department of Neurological Surgery, University of Pittsburgh \\ Medical Center, Pittsburgh, Pennsylvania, United States \\ ${ }^{3}$ Department of Otolaryngology, University of Pittsburgh Medical \\ Center, Pittsburgh, Pennsylvania, United States \\ Address for correspondence Charit Taneja, MD, Division of \\ Endocrinology and Metabolism, University of Pittsburgh Medical \\ Center, Pittsburgh, Pennsylvania, United States \\ (e-mail: tanejac@upmc.edu).

\begin{abstract}
Keywords

- pituitary apoplexy

- COVID-19

- endonasal surgery

This report describes a case of pituitary apoplexy with rapidly evolving hemorrhage in a 74-year-old female with coronavirus disease 2019 (COVID-19) disease. The patient presented with severe headache and mild respiratory symptoms, with laboratories concerning for pituitary hypofunction. Brain imaging demonstrated a sellar mass concerning for a pituitary adenoma with ischemic apoplexy. She subsequently developed visual deficits within 24 hours of presentation, and repeat imaging demonstrated evolving hemorrhage and new mass effect on the optic chiasm. She was successfully managed with urgent endoscopic endonasal surgery despite her COVID-19 positive status by taking special intraoperative precautions to mitigate SARS-CoV2 transmission risk. Only a handful of cases of pituitary apoplexy have been reported in association with COVID-19 disease, and even fewer reports exist of endonasal procedures in such cases. We discuss the potential implication of COVID-19 in the occurrence of pituitary apoplexy, in addition to the safety and success of endonasal surgery in this population.
\end{abstract}

\section{Introduction}

Pituitary apoplexy is an uncommon condition resulting from ischemia or hemorrhage in a pituitary adenoma, or rarely in a physiologically enlarged pituitary gland. It is estimated that this condition affects approximately 2 to $12 \%$ of all patients with pituitary adenomas, most of which are nonfunctioning adenomas. ${ }^{1-3}$ The pathophysiology of pituitary apoplexy is not well defined, but associations have been made with major surgery, hypertension, and dynamic endocrine testing. Additionally, an association has been noted with states that alter the coagulation pathways, including but not limited to pregnancy, coagulopathies, use of anticoagulants or thrombolytic agents, and hemorrhagic infections such as dengue and leptospirosis. ${ }^{4}$ There is often (though not always) an identifiable precipitating factor or condition. The most common presentation is the sudden onset of severe headache; it can be associated with rapidly progressing visual deficits, nausea, pituitary hormone deficiencies, and signs of intracranial hypertension. Management varies by severity of clinical presentation, often requiring surgical decompression received

February 4, 2021 accepted after revision September 20, 2021
DOI https://doi.org/ 10.1055/s-0041-1742104. ISSN 2193-6358.

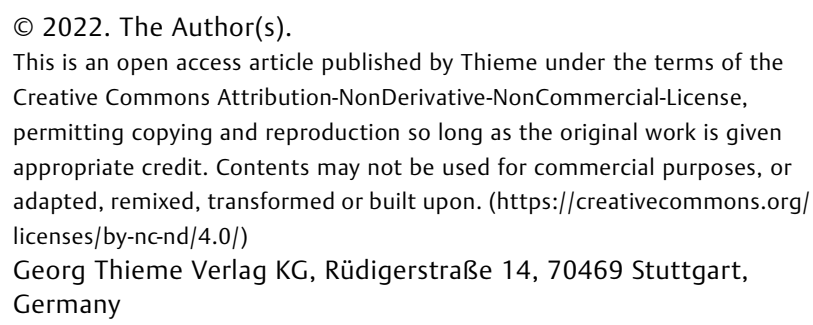


in patients with severe visual or neurologic symptoms. ${ }^{1,5}$ This article describes a case of evolving pituitary apoplexy in a patient with severe acute respiratory syndrome coronavirus 2 (SARS-CoV-2) infection, treated via endoscopic endonasal resection of the adenoma. Given the rarity of the occurrence, the existing literature does not report a distinct association between coronavirus disease 2019 (COVID-19) and pituitary apoplexy. We postulate based on this case report and others in the literature that a variety of pathophysiologic mechanisms could play a role in the development of pituitary apoplexy in such cases, most significantly the hypercoagulable state associated with COVID-19 infection, the exaggerated immune response, and the burden of an acute critical illness.

As the COVID-19 pandemic is continuously evolving, all of the potential effects of this viral infection are not completely understood at this time. We describe this case not only to report its occurrence, but also to discuss the management approach undertaken by our team and the surgical implications in a patient with COVID-19.

\section{Case}

A 74-year-old woman presented with acute onset of severe headache that started approximately 12 hours prior to presentation in the emergency room. She described it as the worst headache of her life, and it was associated with nausea and vomiting. Additionally, she endorsed mild cough, malaise, poor appetite, and a few episodes of diarrhea over the week prior to presentation. She denied any visual complaints, focal weakness, or sensory changes at the time of presentation. Her medical history was significant for multiple sclerosis in remission, hypertension, and hyperlipidemia. Review of symptoms was negative for fever, dyspnea, and loss of smell or taste. On closer questioning, the patient admitted to mild respiratory symptoms approximately 1 week prior to her presentation. Physical exam was notable for a hemodynamically stable patient who was actively vomiting and in moderate distress. There were no focal neurologic deficits and bilateral visual fields were intact on repeat testing. She did not have delayed relaxation of deep tendon reflexes and there was no evidence of dry skin or hair.

Initial imaging with computed tomography scan showed no acute hemorrhage or infarction, but demonstrated a sellar mass warranting further investigation. Pituitary magnetic resonance imaging (MRI) confirmed the sellar mass with suprasellar extension, with complete absence of enhancement and no significant evidence of hemorrhage, slightly abutting the optic chiasm, concerning for a pituitary adenoma with ischemic apoplexy (-Fig. 1). Laboratory studies on admission were notable for a random serum cortisol of $3 \mu \mathrm{g} /$ $\mathrm{dL}$ and an ACTH (adrenocorticotropic hormone) level of $9 \mathrm{pg} /$ $\mathrm{mL}$, which in the setting of acute illness raised the possibility of secondary adrenal insufficiency. Serum prolactin, growth hormone, and thyroid-stimulating hormone were within the normal range; luteinizing hormone $(0.5 \mathrm{mIU} / \mathrm{mL})$ and follicle-stimulating hormone ( $\mathrm{FSH} ; 4.1 \mathrm{mIU} / \mathrm{mL}$ ) were inappropriately low for the postmenopausal age reference range.

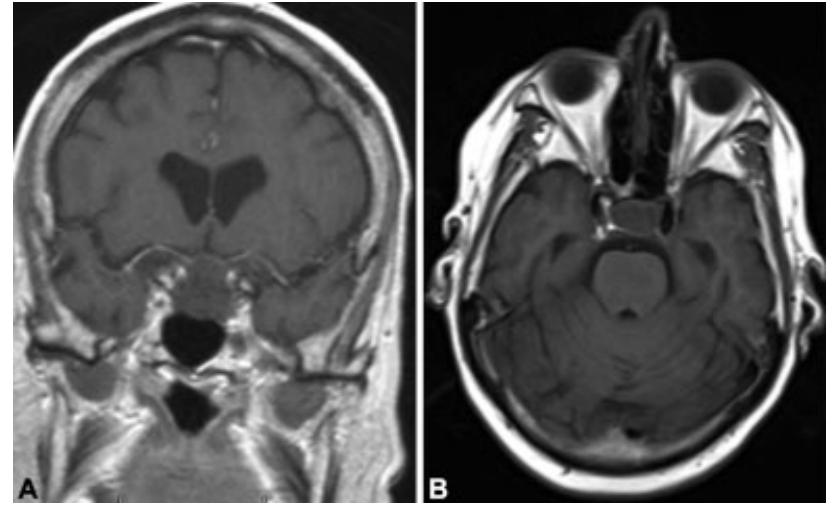

Fig. 1 Coronal (A) and axial (B) MRI demonstrating a poorly enhancing pituitary macroadenoma with suprasellar extension. The lack of enhancement combined with patient symptoms is suggestive of ischemic apoplexy without overt hemorrhage. MRI, magnetic resonance imaging.

Free thyroxine was noted to be low at $0.61 \mathrm{ng} / \mathrm{dL}(0.89-$ $1.78 \mathrm{ng} / \mathrm{dL}$ ). Results of hormonal testing are listed in - Table 1. Serum sodium was noted to be low at $133 \mathrm{mMol} / \mathrm{L}$, urine osmolality was $473 \mathrm{mOsm} / \mathrm{kg}$, and urine sodium was $192 \mathrm{mMol} / \mathrm{L}$. Hyponatremia worsened (serum sodium $127 \mathrm{mMol} / \mathrm{L}$ ) after receiving isotonic fluids in the emergency department, suggesting the possibility of fluid retention in the setting of adrenal insufficiency, hypothyroidism, and/or SIADH (syndrome of inappropriate antidiuretic hormone secretion). Polymerase chain reaction of the nasopharyngeal swab conducted on admission detected SARS-CoV-2. Given the absence of any significant neurologic or visual deficits on presentation, she was admitted to the designated COVID intensive care unit for further observation as opposed to urgent surgical intervention. She was started on stress dose corticosteroids (hydrocortisone $50 \mathrm{mg}$ intravenous every 8 hours) for presumed secondary adrenal insufficiency.

She was minimally symptomatic for COVID-19 and did not require any active interventions to manage the viral illness. The following day, the patient complained of worsening

Table 1 Hormone levels at presentation

\begin{tabular}{|l|l|l|}
\hline Test & Result & Reference range \\
\hline ACTH & 9 & $9-46 \mathrm{pg} / \mathrm{mL}$ \\
\hline Cortisol & 3 & $2-14 \mu \mathrm{g} / \mathrm{dL}^{\mathrm{a}}$ \\
\hline Prolactin & 2.2 & $0.6-20 \mathrm{ng} / \mathrm{mL}$ \\
\hline TSH & 1.543 & $0.3-5.0 \mu \mathrm{IU} / \mathrm{mL}$ \\
\hline Free thyroxine & 0.61 & $0.89-1.78 \mathrm{ng} / \mathrm{dL}$ \\
\hline Growth hormone & 0.77 & $0.01-8.00 \mathrm{ng} / \mathrm{mL}$ \\
\hline LH & 0.5 & $9.0-52.3 \mathrm{mIU} / \mathrm{mL}^{\mathrm{b}}$ \\
\hline FSH & 4.1 & $14.9-124.3 \mathrm{mIU} / \mathrm{mL}^{\mathrm{b}}$ \\
\hline IGF-1 & 45 & $34-245 \mathrm{ng} / \mathrm{mL}$ \\
\hline
\end{tabular}

Abbreviations: ACTH, adrenocorticotropic hormone; FSH, follicle-stimulating hormone; IGF-1, insulin-like growth factor 1; LH, luteinizing hormone; TSH, thyroid-stimulating hormone.

${ }^{a}$ Sample obtained between 4 and 6 pm.

${ }^{\mathrm{b}}$ For postmenopausal females. 


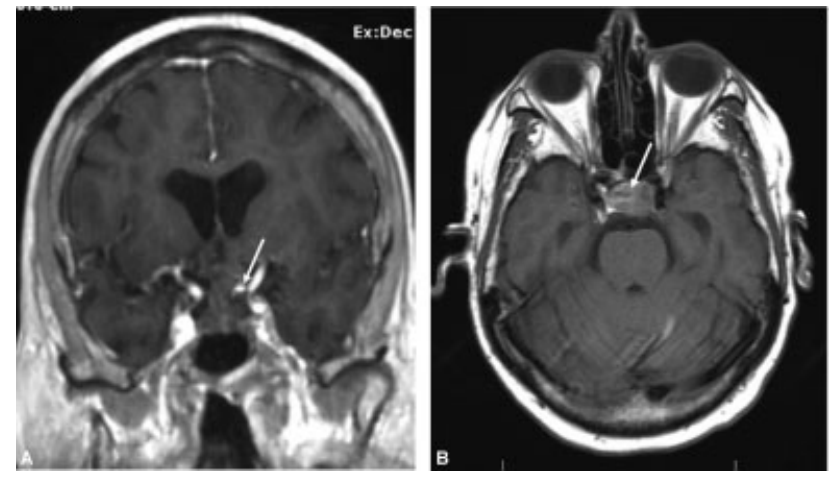

Fig. 2 Coronal (A) and axial (B) MRIs obtained after worsening of patient symptoms demonstrate interval ( $<24$ hours) hemorrhage into the tumor (arrow). MRI, magnetic resonance imaging.

headache and decreased visual acuity in both eyes. She reported blurry peripheral vision that progressively worsened, especially in the left eye. Repeat MRI of the pituitary redemonstrated the sellar mass. Increased heterogeneous, ill-defined areas of $\mathrm{T} 1$ hyperintensity consistent with evolving hemorrhage and new upward mass effect on the optic chiasm greater on the left side were noted ( - Fig. 2). Repeat formal ophthalmologic testing showed new bitemporal visual field defects $(\mathrm{L}>\mathrm{R})$ and a central bitemporal visual defect on the left side. These findings corresponded with the left optic nerve compression at the level of the posterior chiasm seen on MRI. Given her progression and minimal COVID-19 symptomatology, she consented to urgent surgical decompression via an endoscopic endonasal approach (EEA) for resection of the pituitary adenoma.

The patient was taken urgently for endoscopic endonasal surgery in a designated COVID room (negative pressure, 32 air exchanges/hour). The entire surgical, anesthesia, and nursing team utilized appropriate personal protective equipment (PPE) including N95 masks, eye protection, and gowning. Intraoperative COVID-19 precautions including a tracheal suction placed in the nasopharynx throughout surgery, additional standard suction tip used during drilling (two-surgeons, four-hands technique), and protective nasal sleeves (SPIWay) were employed to reduce aerosolization. Personnel within the operating room were limited to a single anesthesia provider, a surgical technologist, a circulating nurse, and two attending surgeons. The total surgical time was approximately 90 minutes despite a relatively firm tumor. A surgical runner to provide any needed additional equipment was located immediately outside of the operating room. Neurophysiological monitor was utilized with extended wires to allow the technologist to be outside of the operating room except during lead insertion. Similarly, patient transfers were minimized and limited to the intensive care unit and operating room.

Surgical pathology showed large areas of devitalized tissue consistent with an apoplectic pituitary adenoma, with the $\mathrm{K}_{\mathrm{i}}-67$ proliferative index of approximately 1 to $2 \%$. Immunohistochemistry demonstrated positive staining for FSH, synaptophysin and GATA3 in viable cells, and weakly positive staining for estrogen receptors.
She recovered well from the surgery, with marked improvement in vision as well as her headache and associated symptoms. Her serum sodium levels normalized and fluids were liberalized. She had no respiratory sequelae from general anesthesia and did not require any adjunct treatment for COVID-19. She was discharged to home on a physiologic replacement dose of steroids, 6 days after the surgery with quarantine precautions, and she was subsequently started on thyroid hormone replacement with levothyroxine for a persistently low free thyroxine level. No personnel involved with the surgical case developed symptoms of COVID-19 or had a positive test for SARS-CoV-2 in the subsequent 3 weeks.

\section{Discussion}

December 2019 marked the beginning of the COVID-19 pandemic caused by the SARS-CoV-2 virus. After a year of scientific discoveries and advances in the medical field related to this disease, we are still uncovering new manifestations and implications of this grave respiratory illness. This case raises the question whether the presence of SARS-CoV-2 in this patient was responsible for or contributed to the development of apoplexy within the pituitary adenoma, especially given the vasculopathy observed in some COVID-19 patients. ${ }^{6}$ In addition, this report highlights the feasibility and success of emergent neurosurgical intervention despite the challenging scenario.

There have been several reports of a possible association of COVID-19 with an immune response-related hypercoagulable state and an increased risk of cerebrovascular events, although the risk may be similar to other viral infections..$^{7-10}$ SARS-CoV-2 may affect the coagulation cascade by inducing thrombocytopenia and platelet dysfunction, and in combination with overstimulation of the pituitary gland in the setting of an acute infection, this could potentially precipitate pituitary infarction and/or hemorrhage. ${ }^{11}$ Additionally, there are data linking COVID-19 and neurological problems, even in patients with mild cases of the infection. There are some reports suggesting that the SARS-CoV-2 virus can reach the brainstem and neural tissues via two potential routes; either by hematogenous spread or through the nasopharyngeal epithelium and the olfactory nerve. Moreover, it is possible that the virus has special affinity for neural tissues due to the expression of ACE2 receptors in the cerebral vascular endothelium, which are binding sites for the virus. ${ }^{12}$ One must also note that pituitary apoplexy is often reported following an inciting event or illness (cardiac or orthopedic surgery, critical illness), and severe presentations of COVID19 could certainly represent such an inciting event, though our patient only had a mild respiratory illness. ${ }^{6,11}$

There have been at least three prior case reports demonstrating pituitary apoplexy in SARS-CoV-2-positive patients, one of which was in a pregnant female. ${ }^{13-15}$ It is interesting to note that two of these patients were minimally symptomatic from COVID-19 similar to our patient, whereas the third patient had a severe respiratory illness and eventually died from refractory hypoxemia. Whether COVID-19 had a causal role to play in the development of 
pituitary apoplexy or if it was a mere coincidence still remains an unanswered question.

The pregnant woman in the first report was initially managed medically, and surgery was delayed for about 7 days to allow for safe delivery of the baby. ${ }^{13}$ She then underwent successful endoscopic transsphenoidal decompression of the mass with strict COVID-19 precautions in the operating room such as multiple layers of PPE, powered air-purifying respirators, decreased number of personnel, and delays after intubation and extubation. The patients in the other two reports did not undergo neurosurgical intervention. One report of a transsphenoidal resection of a pituitary tumor suggests airway management guidelines for such cases, including the use of negative pressure rooms for induction and intubation and plastic drapes covering the patient during intubation. ${ }^{16}$ Early in the pandemic, the Pituitary Society came out with guidance for management of pituitary disease during the pandemic. ${ }^{17}$ They suggest risk-stratifying patients and delaying surgery for COVID-19-positive patients by 2 or more weeks when possible. When there is a need for emergent surgery, they recommend considering alternative transcranial approaches to avoid nasal mucosa, using nonpowered tools in place of high-speed drilling in addition to the use of PPE and respirators. There is, however, no evidence that these precautions are necessary and transcranial approaches are generally outside the standard of care for most pituitary tumors.

Indeed, there is growing evidence for safe endonasal surgery during the COVID pandemic. Aerosolization during endonasal surgery has been examined using cadaveric simulation of splatter patterns using fluorescein solution as a novel way of examining potential contamination with secretions during surgery. While one study reported a significant contamination risk during endonasal procedures using highspeed drilling, similar experiments repeated at our institution did not reveal significant contamination of the surgical field with drilling. ${ }^{18,19}$ The use of constant suctioning during surgery and avoiding removing the moving drill from the nasal cavity are potential ways to mitigate aerosolization risk, droplet spread, and the resultant contamination. ${ }^{19-22}$

The patient in this case report required emergent neurosurgical intervention given her rapidly progressive visual deficits as well as progression of hemorrhagic changes on pituitary imaging. Our team opted to use a standard EEA despite the COVID-19-positive status of the patient. Mitigation strategies used in this case included a nasopharyngeal suction in addition to standard suction tips used during dissection and powered instrumentation, limiting the length of surgery and minimizing personnel exposure to the patient. Transmissibility of this particular patient is unclear given the length of time (over 1 week) since her symptomatic period, but this case and others demonstrate the potential for safe endonasal surgery, regardless of COVID status.

In conclusion, the possibility of pituitary apoplexy occurring as a sequela of COVID-19 infection should be considered. The pathogenesis and causality of this occurrence remains questionable and deserves further investigation, but could be hypothesized to be related to an immune-related hypercoagulable state. Additionally, this case demonstrates the need for and feasibility of emergent endonasal surgery in appropriately selected cases.

Availability of Data and Material

Data sharing not applicable to this article as no datasets were generated or analyzed during the current study.

Code Availability

Not applicable.

Funding

None.

Conflict of Interest

None declared.

\section{References}

1 Singh TD, Valizadeh N, Meyer FB, Atkinson JL, Erickson D, Rabinstein AA. Management and outcomes of pituitary apoplexy. J Neurosurg 2015;122(06):1450-1457

2 Wildemberg LE, Glezer A, Bronstein MD, Gadelha MR. Apoplexy in nonfunctioning pituitary adenomas. Pituitary 2018;21(02): 138-144

3 Briet C, Salenave S, Bonneville J-F, Laws ER, Chanson P. Pituitary Apoplexy. Endocr Rev 2015;36(06):622-645

4 Tan SK, Seow CJ, Tan E, Chau YP, Dalan R. Pituitary apoplexy secondary to thrombocytopenia due to dengue hemorrhagic fever: a case report and review of the literature. Endocr Pract 2014;20(04):e58-e64

5 Capatina C, Inder W, Karavitaki N, Wass JA. Management of endocrine disease: pituitary tumour apoplexy. Eur J Endocrinol 2015;172(05):R179-R190

6 Siddiqi HK, Libby P, Ridker PM. COVID-19-a vascular disease. Trends Cardiovasc Med 2021;31(01):1-5

7 Li Y, Li M, Wang M, et al. Acute cerebrovascular disease following COVID-19: a single center, retrospective, observational study. Stroke Vasc Neurol 2020;5(03):279-284

8 Shahjouei S, Naderi S, Li J, et al. Risk of stroke in hospitalized SARSCoV-2 infected patients: a multinational study. EBioMedicine 2020;59:102939

9 Mazzeffi MA, Chow JH, Tanaka K. COVID-19 associated hypercoagulability: manifestations, mechanisms, and management. Shock 2021;55(04):465-471

10 Kichloo A, Dettloff K, Aljadah M, et al. COVID-19 and hypercoagulability: a review. Clin Appl Thromb Hemost 2020;26:1076029620962853

11 Frara S, Allora A, Castellino L, di Filippo L, Loli P, Giustina A. COVID19 and the pituitary. Pituitary 2021;24(03):465-481

12 Chigr F, Merzouki M, Najimi M. Autonomic brain centers and pathophysiology of COVID-19. ACS Chem Neurosci 2020;11(11): $1520-1522$

13 Chan JL, Gregory KD, Smithson SS, Naqvi M, Mamelak AN. Pituitary apoplexy associated with acute COVID-19 infection and pregnancy. Pituitary 2020;23(06):716-720

14 Solorio-Pineda S, Almendárez-Sánchez CA, Tafur-Grandett AA, et al. Pituitary macroadenoma apoplexy in a severe acute respiratory syndrome-coronavirus-2-positive testing: causal or casual? Surg Neurol Int 2020;11:304

15 Ghosh R, Roy D, Roy D, et al. A rare case of SARS-CoV-2 infection associated with pituitary apoplexy without comorbidities. J Endocr Soc 2021;5(03):a203

16 e Santos CdS, da Costa Lima Filho LM, Santos CAT, Neill JS, Vale HF, Kurnutala LN. Pituitary tumor resection in a patient with SARSCoV-2 (COVID-19) infection. A case report and suggested airway management guidelines. Braz J Anesthesiol 2020;70(02):165-170 
e12 Rapidly Progressive Pituitary Apoplexy in a Patient with COVID-19 Taneja et al.

17 Fleseriu M, Buchfelder M, Cetas JS, et al. Pituitary society guidance: pituitary disease management and patient care recommendations during the COVID-19 pandemic-an international perspective. Pituitary 2020;23(04):327-337

18 Workman AD, Welling DB, Carter BS, et al. Endonasal instrumentation and aerosolization risk in the era of COVID-19: simulation, literature review, and proposed mitigation strategies. Int Forum Allergy Rhinol 2020;10(07):798-805

19 Snyderman $\mathrm{CH}$, Gardner PA. Endonasal drilling may be employed safely in the COVID-19 era. Int Forum Allergy Rhinol 2020;10(09): 1118-1119
20 Workman AD, Xiao R, Feng A, et al. Suction mitigation of airborne particulate generated during sinonasal drilling and cautery. Int Forum Allergy Rhinol 2020;10(10):1136-1140

21 Dharmarajan H, Freiser ME, Sim E, et al. Droplet and aerosol generation with endonasal surgery: methods to mitigate risk during the COVID-19 pandemic. Otolaryngol Head Neck Surg 2021;164(02):285-295

22 Velasquez N, Ahmed $\mathrm{OH}$, Lavigne P, et al. Utility of nasal access guides in endoscopic endonasal skull base surgery: assessment of use during cadaveric dissection and workflow analysis in surgery. J Neurol Surg B Skull Base 2021;82(05):540-546 